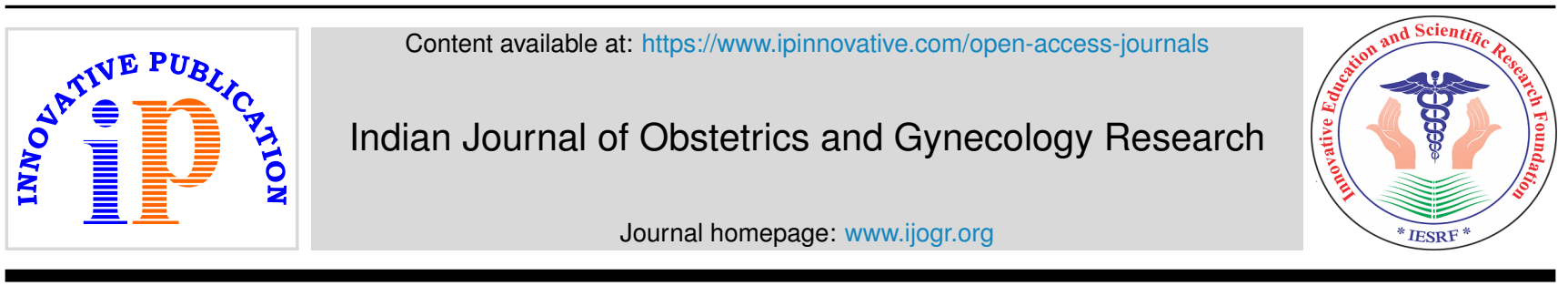

Original Research Article

\title{
Efficacy of mifepristone followed by misoprostol in the first and second trimester MTP in 2019
}

\author{
Mugunthan Karalmarks ${ }^{1} *$, K Kohila ${ }^{1}$, Jayanthi ${ }^{1}$ \\ ${ }^{1}$ Dept. of Obstetrics and Gynaecology, Saveetha Medical College \& Hospital, Chennai, Tamil Nadu, India
}

\section{A R T I C L E I N F O}

\section{Article history:}

Received 12-08-2020

Accepted 07-09-2020

Available online 07-12-2020

\section{Keywords:}

Abortion

Dilatation and curettage (D\&C)

Hysterotomy

MTP (Medical Termination of

Pregnancy)

\begin{abstract}
A B S T R A C T
Objective: To determine the efficacy of mifepristone followed by misoprostol in the first and second trimester.

Materials and Methods: A retrospective study is done to evaluate the efficacy of mifepristone followed by misoprostol in the first and second trimester of medical termination of pregnancy using the medical records from January 2019 to December 2019 in Saveetha medical college and hospital. The efficacy is determined by the completion of abortion, need for D\&C and the need for hysterectomy after the medical abortion. And the analysis and evaluation of data is done using the SPSS software.

Result: The study demonstrates that the use of mifepristone followed by misoprostol has got good efficacy in the first trimester and second trimester, and also shows that it's efficacy is better in the first trimester compared to the second trimester.

(C) This is an open access article distributed under the terms of the Creative Commons Attribution License (https://creativecommons.org/licenses/by/4.0/) which permits unrestricted use, distribution, and reproduction in any medium, provided the original author and source are credited.
\end{abstract}

\section{Introduction}

\subsection{Abortion}

Defined as the expulsion of the fetus or the products of conception before the period of viability.

An abortion can be spontaneous or induced.

Induced abortions or also called the Medical termination of pregnancy(MTP) is done for the following reasons:

1. Therapeutic

2. Eugenic.

3. Humanitarian

4. Social

5. Environmental

\subsection{Medical abortion}

Use of medication to end the pregnancy, it does not require any surgery or anaesthesia and can be done in clinic or at home with follow up.

\footnotetext{
* Corresponding author.

E-mail address: mugunthanmarx@gmail.com (M. Karalmarks).
}

The procedure is done by using the following medications: ${ }^{1}$

1. Oral mifepristone and oral misoprostol.

2. Oral mifepristone and vaginal, buccal or sublingual misoprostol.

3. Methotrexate and vaginal misoprostol.

4. Vaginal misoprostol alone.

The duration of pregnancy (gestation duration) is divided into three trimesters: first trimester(14 weeks of gestation since the first day of the last menstrual period), second trimester(14-28 weeks), third trimester(28 weeks to delivery).First trimester surgical abortion (up to 12 or 14 weeks) and medical abortion (up to nine weeks) should be performed as outpatient procedures.

Best method of MTP:

1. $<7$ weeks - Medical abortion

2. 7-15 weeks - suction evacuation

3. $>15$ weeks - prostaglandins

Although the medical abortion is most commonly used up to 63 days of gestation, the treatment also is effective 
after 63 days of gestation. There is a increase in second trimester abortion because of increased determination of the sex linked genetic, metabolic disorders.

Mifepristone ${ }^{2}$ it's a derivative of norethindrone which binds to the progesterone receptor and has a affinity greater than progesterone itself but does not activate the receptor, thereby acting as an antiprogestin, it blocks the progesterone receptors causes estrogen dominance and results in intrauterine fetal death. It also sensitizes the uterus to the activity of the prostaglandin. The uterine contractility does not increase until 24-36 hours after mifepristone administration.

Misoprostol, a synthetic prostaglandin E1 has proven as an abortifacient since 1987. It is stable at room temperature, requires no refrigeration, is cost effective, has fewer side effects, is a potent uterotonic and cervical ripening agent. It can be used by both the oral as well as vaginal route and also used along with other drugs. If mifepristone is given prior to induction with misoprostol, there is disruption of pregnancy causing decidual necrosis, myometrial contractions, and cervical softening resulting in earlier second trimester abortion. The most commonly used dose of mifepristone is 200 milligrams is given on day 1 and followed by which after $36-48$ hours misoprostol 600 micrograms is administered. ${ }^{3,4}$

Thus, a combination of the two can significantly improve the efficacy of the misoprostol for the termination of second trimester termination of the pregnancy.

\section{Materials and Methods}

A retrospective study done using the medical records of 100 patients who have undergone the medical abortion procedure in Saveetha hospital. The Data were collected from the medical records of the hospital from January 2019 to December 2019 at the department of obstetrics and gynaecology, Saveetha medical college and hospital. The study analysis the efficacy of the medical abortion using the mifepristone(200 milligram) followed by misoportol (600 microgram) based the following criteria : 1) completion of the abortion 2) need for dilatation and curettage 3 ) and the need for hysterotomy after the medical abortion is undergone. The case study included 100 patients enrolled, out of which the patients were grouped into those in the first trimester who have undergone the MTP and those in the second trimester who have undergone the MTP. The assessment of the efficacy of the drugs is done based on the completion of the abortion and the well being of the patient after the procedure. The completion of the abortion without the requirement of any other intervening procedure concludes the better efficacy of the drugs and the procedure.

\section{Result}

Out of the 100 cases in our study, 50 of them were in the first trimester (between 7 weeks to 14 weeks) and had undergone the medical abortion, and the other 50 of them who were in their second trimester(between 14-20 weeks patients were common) had also undergo medical abortion. Patients who have undergone surgery as the primary procedure and people with hypersensitive to prostaglandins or in whom this group of drugs was contraindicated, severe anemia $(\mathrm{Hb}<5 \mathrm{~g} \%)$, low-lying placenta, scarred uterus, coagulation disorder, current uncontrolled hypertension, cardiac disorder, jaundice or renal disease are excluded. ${ }^{5}$

The data collection done in our institution revealed the following:(Figure 1)

1. Total number of cases collected: 100

2. Cases of first trimester: 50

3. Cases of second trimester: 50

\section{No. of Patients}

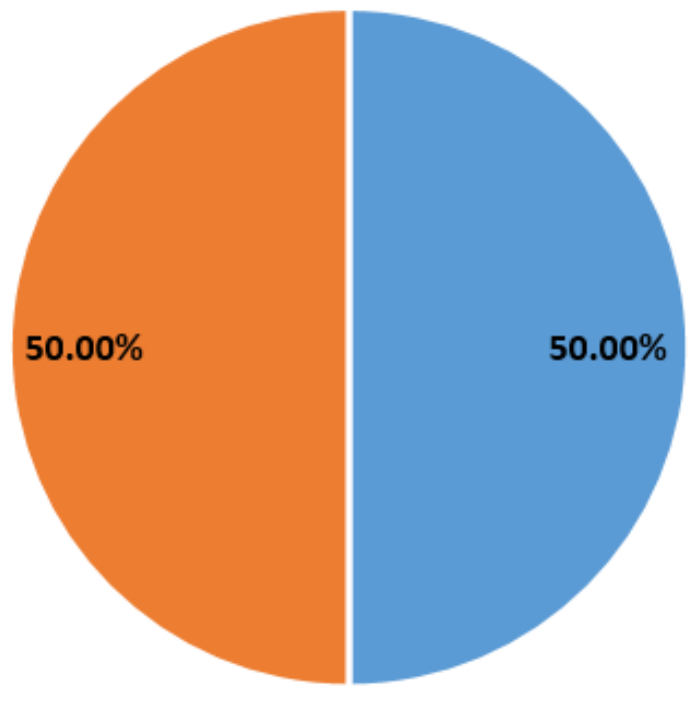

- First Trimester $\quad$ Second Trimester

The study conducted demonstrated that out of the 100 patients who have had undergone medical abortion using the drugs mifepristone followed by misoprotol 76 of them had complete abortions without the need any intervening procedure after the medical abortion. That is the patients didn't require any dilatation and curettage or hysterotomy after the medical abortion. Evaluation of the medical record of the patients also revealed that out the 50 first trimester patients who have under abortion 42 of the patients had a successful completion of abortion but the other 8 of the 
first trimester patients had to under go for dilatation and curettage.

Similarly, other 50 patients of the second trimester who had undergone abortion 34 out of them had complete abortion, but 16 of the patients did not. Thus the 16 patients had to undergo other procedures for complete abortion, that is 14 out of them had undergone dialation and curettage and 2 out of the 16 had gone hysterotomy. Hence our study showed that the efficacy of mifepristone followed by misoprostol is good both in the first and second trimester, it also shows that the efficacy is better in the first trimester patients compared to that of the second trimester patients (Figure 2).

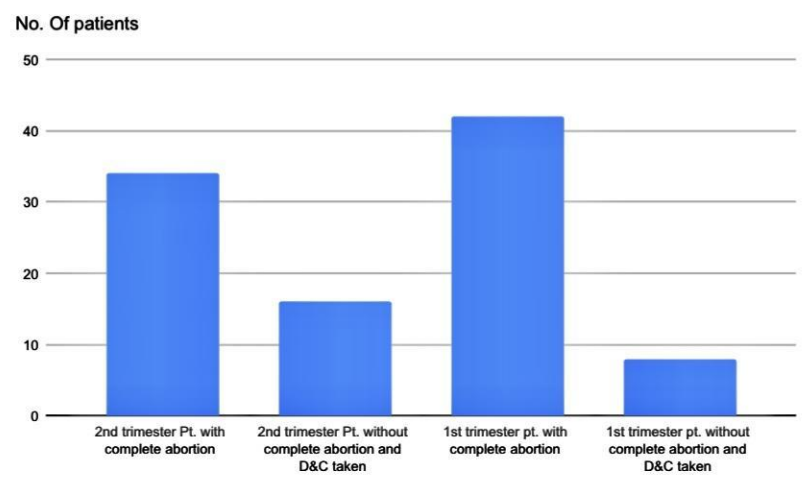

Fig. 2:

\section{Discussion}

In our study we have shown that the medical abortion using mifepristone followed by misoprotol has got good efficacy and it also shows that the efficacy was found to better in the first trimester patients than the second trimester patients. Similarly, a systematic review published in 2007 found that the efficacy of misoprostol regimens at gestational ages $\leq 63$ days (first trimester) ranged from $84 \%$ to $96 \%$. The World Health Organization (WHO) and Royal College of Obstetrics \& Gynecology (RCOG) strongly recommend the use of the anti-progestin and mifepristone, followed by misoprostol, as the medical method for secondtrimester abortion. There are other studies which also show the efficacy of this regimen over the mono therapy one such study states that Gestational sac expulsion rates were $84 \%$ versus $67 \%$ (mifepristone-misoprostol vs. misoprostol monotherapy; relative risk, 1.2). Surgical aspiration was less likely in the mifepristone-misoprostol group than the misoprostol group. Pre-treatment of misoprostol with mifepristone significantly increased the complete abortion rate and, hence, reduced the need for surgical evacuation. ${ }^{6}$ Studies have shown that pretreatment with mifepristone makes it possible to conduct midtrimester abortion on a daycare basis. Second Trimester Abortions in India by
Suchitra S Dalvie have shown that the use of this regimen in the second trimester is recent times have increased due to its ease of use and the efficacy even though some of the patients required surgery after this most of them had completely abortion with this regimen. ${ }^{7}$ The other advantage of the regimen is that the effectiveness of the drug mifepristone doesn't seem to be affected by the dose, and has been proven by various studies that the administration of 200 $\mathrm{mg}$ instead of $600 \mathrm{mg}$ followed by misoprostol have similar outcomes. This thereby shows that the efficacy of the regimen mifepristone followed by the misoprostol has better efficacy and outcome compared to the other therapies. ${ }^{8}$ As the results obtained from our study relates to the results of previous studies and also share some similarities between them, which states that there are only few patients who required surgery after this medical abortion and many had compete abortion without the need of any dilatation and curettage or hysterotomy. The evaluation of the medical records have shown that 76 who have undergone medial abortion had an completely normal and health life without the need of any other procedure but out of that 8 in first trimester and 16 in second trimester have under gone dilatation and curettage. Therefore, this has been accredited with the report that the efficacy of mifepristone followed by misoprostol in the first and second trimester had got good results and prognosis. While the efficacy of this regimen seems to have a better efficacy in the first trimester than the second trimester.

\section{Conclusion}

Medical abortion is a major social and health issue, particularly in the developing countries. Thus the need for safe and effective regimen has increased in recent times, and in the recent times it's shown that mifepristone followed by misoprostol is a safe and effective regimen in the first and second-trimester abortion. Our study has shown that the efficacy of the regimen i.e; mifepristone followed misoprostol have a better efficacy rate in Bothe the trimester and also shows that comparatively that it's efficacy is better in the first trimester. Thus we conclude the study by stating that the efficacy of the regimen is better in first trimester patients.

\section{Source of Funding}

None.

\section{Conflict of Interest}

The authors declare no conflict of interest.

\section{References}

1. Lalitkumar S, Bygdeman M, Gemzell-Danielsson K. Mid-trimester induced abortion: a review. Hum Reprod Update. 2007;13(1):37-52. do1:10.1093/humupd/dmI049. 
2. Wildschut H, Both MI, Medema S, Thomee E, Wildhagen MF, Kapp N. Medical methods for mid-trimester termination of pregnancy. Cochrane Database Syst Rev. 2011;19(1):CD005216. 10ن$10.1002 / 14651858 . C D 005216 . \mathrm{pub2}$.

3. Safe abortion: technical and policy guidance for health systems. Second edition ed. World Health Organization; 2012.

4. Kulier R, Kapp N, Gülmezoglu AM, Hofmeyr GJ, Cheng L, Campana A, et al. Medical methods for first trimester abortion. 2011 do1:10.1002/14651858.CD002855.pub4.

5. Ngoc NT, Scochet T, Raghavan S, Blum J, Nga NTB, Minh NTH. Mifepristone and misoprostol compared with misoprostol alone for second-trimester abortion: a randomized controlled trial. Obstet Gynaecol. 2011;118(3):601-8.

6. Garg G, Takkar N, Sehgal A. Buccal Versus Vaginal Misoprostol Administration for the Induction of First and Second Trimester Abortions. $\quad J$ Obstet Gynecol India. 2015;65(2):111-6. dol:10.1007/s [3224-(0)4-(0605-5].

7. Hou S, Zhang L, Chen Q, Fang A, Cheng L. One and two day mifepristone- misoprostol intervals for second trimester termination of pregnancy between 13 and 16 weeks of gestation. Int J Obstet Gynaecol. 2010;111(2):126-30.

8. World Health Organization (WHO) Unsafe abortion: global and regional estimates of the incidence of unsafe abortion and associated mortality in 2008. 6. Geneva, Switzerland: World Health Organization; 2011.

\section{Author biography}

K Kohila, Associate Professor

Cite this article: Karalmarks M, Kohila K, Jayanthi. Efficacy of mifepristone followed by misoprostol in the first and second trimester MTP in 2019. Indian J Obstet Gynecol Res 2020;7(4):544-547. 